\title{
Construction of Solutions for Nonintegrable Systems with the Help of the Painlevé Test
}

\author{
S.Y. Vernov \\ Skobeltsyn Institute of Nuclear Physics, Moscow State University, \\ Vorob'evy Gory, Moscow, 119992, Russia. \\ svernov@theory.sinp.msu.ru
}

\begin{abstract}
The generalized Hénon-Heiles system with an additional nonpolynomial term has been considered. In two nonintegrable cases with the help of the Painlevé test new special solutions have been found as converging Laurent series, depending on three parameters. For some values of these parameters the obtained Laurent series coincide with the Laurent series of the known elliptic solutions. The calculations have been made with use of computer algebra system REDUCE. The obtained local solutions can assist to find the elliptic three parameters solutions. The corresponding algorithm has been realized in REDUCE and Maple.
\end{abstract}

\section{The Painlevé Test}

When we study some mechanical problem time is assumed to be real, whereas the integrability of motion equations is connected with the behavior of their solutions as functions of complex time. Solutions of a system of ODE's are regarded as analytic functions, maybe with isolated singular points. A singular point of a solution is said critical (as opposed to noncritical) if the solution is multivalued (single-valued) in its neighborhood and movable if its location depends on initial conditions. The general solution of an ODE of order $N$ is the set of all solutions mentioned in the existence theorem of Cauchy, i.e. determined by the initial values. It depends on $N$ arbitrary independent constants. A special solution is any solution obtained from the general solution by giving values to the arbitrary constants. A singular solution is any solution which is not special, i.e. which does not belong to the general solution. A system of ODE's has the Painlevé property if its general solution has no movable critical singularity point 1 .

The Painlevé test is any algorithm, which checks some necessary conditions for a differential equation to have the Painlevé property. The original algorithm, developed by P. Painlevé and used by him to find all the second order ODE's with Painlevé property, is known as the $\alpha$-method. The method of S.V. Kovalevskaya [2] is not as general as the $\alpha$-method, but much more simple. The remarkable property of this test is that it can be checked in a finite number of steps. In 1980, motivated by the work of S.V. Kovalevskaya [2], M.J. Ablowitz, A. Ramani and H. Segur [3] developed a new algorithm of the Painlevé test for ODE's. This algorithm appears very useful to find solutions as a formal 
Laurent series. First of all, it allows to determine the dominant behavior of a solution in the neighborhood of the singularity point $t_{0}$. If the solution tends to infinity as $\left(t-t_{0}\right)^{\beta}$, where $\beta$ is a negative integer number, then substituting the Laurent series expansions one can transform nonlinear differential equations into a system of linear algebraic equations on coefficients of the Laurent series. If a single-valued solution of autonomous system depends on not only the parameter $t_{0}$, which characterizes the syngularity point location, but also on other parameters, then some coefficients of its Laurent series have to be arbitrary and the corresponding systems have to have zero determinants. The numbers of such systems (named resonances or Kovalevskaya exponents) can be determined due to the Painlevé test. In such a way we obtain solutions only as formal series, but for some nonintegrable systems, for example, the generalized Hénon-Heiles system [4], the convergence of the Laurent- and psi-series solutions has been proved. Such solutions also assist to find the elliptic solutions [5].

\section{The Hénon-Heiles Hamiltonian}

Let us consider the generalized Hénon-Heiles system with an additional nonpolynomial term, which is described by the Hamiltonian:

$$
H=\frac{1}{2}\left(x_{t}^{2}+y_{t}^{2}+\lambda x^{2}+y^{2}\right)+x^{2} y-\frac{C}{3} y^{3}+\frac{\mu}{2 x^{2}}
$$

and the corresponding system of the motion equations:

$$
\left\{\begin{array}{l}
x_{t t}=-\lambda x-2 x y+\frac{\mu}{x^{3}}, \\
y_{t t}=-y-x^{2}+C y^{2},
\end{array}\right.
$$

where $x_{t t} \equiv \frac{d^{2} x}{d t^{2}}$ and $y_{t t} \equiv \frac{d^{2} y}{d t^{2}}, \lambda, \mu$ and $C$ are arbitrary numerical parameters. If $C=1, \lambda=1$ and $\mu=0$, then (1) is the initial Hénon-Heiles Hamiltonian [6].

The general solutions in the analytic form are known [7] only in the three integrable cases $(C=-1$ and $\lambda=1 ; C=-6$ and $\lambda$ is an arbitrary number; $C=-16$ and $\lambda=1 / 16$ ), in other cases not only four-, but even three-parameter exact solutions have yet to be found.

In all above-mentioned cases system (2) is integrable at any value of $\mu$. Moreover the function $y$, solution of system (2), satisfies the following fourth-order equation, which does not include $\mu$ :

$y_{t t t t}=(2 C-8) y_{t t} y-(4 \lambda+1) y_{t t}+2(C+1) y_{t}^{2}+\frac{20 C}{3} y^{3}+(4 C \lambda-6) y^{2}-4 \lambda y-4 H$,

where $H$ is the energy of the system. If $x_{0}=C y_{0}^{2}-y_{0}-y_{0 t t} \neq 0$, then $H$ is not an arbitrary parameter, but a function of initial data: $y_{0}, y_{0 t}, y_{0 t t}$ and $y_{0 t t t}$. The form of this function depends on $\mu$.

The Painlevé test of eq. (3) gives the following dominant behaviors and resonance structures: 
1. The function $y$ tends to infinity as $b_{-2}\left(t-t_{0}\right)^{-2}$, where $b_{-2}=-3$ or $b_{-2}=\frac{6}{C}$.

2. For $b_{-2}=-3$ (the Case 1$)$ the values of resonances are $r=-1,10,(5 \pm$ $\sqrt{1-24(1+C)}) / 2$. In the Case $2\left(b_{-2}=\frac{6}{C}\right) r=-1,5,5 \pm \sqrt{1-48 / C}$.

The resonance $r=-1$ corresponds to arbitrary parameter $t_{0}$. Other values of $r$ determine powers of $t$ (their values are $r-2$ ), at which new arbitrary parameters can appear as solutions of the linear systems with zero determinant. For integrability of system (2) all values of $r$ have to be integer and all systems with zero determinants have to have solutions at any values of included in them free parameters. It is possible only in the integrable cases.

For the search for special solutions, it is interesting to consider such values of $C$, for which $r$ are integer numbers either only in Case 1 or only in Case 2. If there exist a negative integer resonance, different from $r=-1$, then such Laurent series expansion corresponds rather to special than general solution. We demand that all values of $r$, but one, are nonnegative integer numbers and all these values are different. From these conditions we obtain the following values of $C: C=-1$ and $C=-4 / 3$ (Case 1 ), or $C=-16 / 5, C=-6$ and $C=-16$ (Case 2, $\alpha=\frac{1-\sqrt{1-48 / C}}{2}$ ), and also $C=-2$, in which these two Cases coincide. It has been shown in [8] (for $\mu=0$ ) and 9] (for an arbitrary value of $\mu$ ) that single-valued three-parameter special solutions can exist only in two nonintegrable cases: $C=-16 / 5$ and $C=-4 / 3$ ( $\lambda$ is arbitrary).

Using the method of construction of the Laurent series solutions for nonlinear differential equations describing in [8], we obtain single-valued local solutions of eq. (3). At $C=-4 / 3$ these solutions are:

$$
y=-3 \frac{1}{t^{2}}+b_{-1} \frac{1}{t}+\frac{29}{24} b_{-1}^{2}+\frac{1}{2} \lambda-\frac{3}{4}+\left(\frac{17}{6} b_{-1}^{2}+\frac{5}{3} \lambda-\frac{5}{4}\right) b_{-1} t+b_{2} t^{2}+\ldots
$$

There exist four possible values of the parameter $b_{-1}$ :

$$
b_{-1}= \pm \sqrt{\frac{105-140 \lambda \pm \sqrt{7\left(1216 \lambda^{2}-1824 \lambda+783\right)}}{385}} .
$$

The parameters $b_{2}$ and $b_{8}$, coefficients at $t^{2}$ and $t^{8}$ correspondingly, are arbitrary. The energy $H$ enters in coefficients beginning from $b_{4}$.

At $C=-16 / 5$ we obtain the following solutions:

$$
y=-\frac{15}{8 t^{-2}}+\tilde{b}_{-1}-\frac{5}{32}+\frac{62}{45} \tilde{b}_{-1}^{2}+\left(\frac{5}{12} \lambda+\frac{632}{225} \tilde{b}_{-1}^{2}-\frac{25}{192}\right) \tilde{b}_{-1} t+\ldots
$$

with

$$
\tilde{b}_{-1}= \pm \frac{3 \sqrt{6872250-21991200 \lambda \pm 52360 \sqrt{35\left(2048 \lambda^{2}-1280 \lambda+387\right)}}}{41888} .
$$

The coefficients $\tilde{b}_{3}$ and $\tilde{b}_{8}$ are arbitrary parameters. Beginning from $\tilde{b}_{4}$ some coefficients include the energy $H$. So, the obtained local solutions depend on 
four independent parameters: $t_{0}, H$ and two coefficients $\left(b_{2}\right.$ and $b_{8}$ or $\tilde{b}_{3}$ and $\left.\tilde{b}_{8}\right)$. With the help of some computer algebra system, for example, REDUCE [12, these solutions can be obtained with arbitrary accuracy. When a formal series is obtained the question about its convergence arises. The convergence of psi-series solutions of the generalized Hénon-Heiles system with $\mu=0$ on some real time interval has been proved in [4].

\section{Global Single-Valued Solutions}

We have found local single-valued solutions. Of course, existence of local singlevalued solutions is necessary, but not sufficient condition to exist global ones, because solutions, which are single-valued in the neighborhood of one singularity point, can be multivalued in the neighborhood of another singularity point. So, we can only assume that global three-parameter solutions are single-valued. If we assume this and moreover that these solutions are elliptic functions (or some degenerations of them), then we can seek them as solutions of some polynomial first order equations. There are a few methods to construct such solutions, representing them as the finite Taylor or Laurent series of elliptic functions or degenerate elliptic functions, for example, $\tanh (t)$. These methods use results of the Painlevé test, but don't use the obtained Laurent-series solutions. In 2003 R. Conte and M. Musette 5 have proposed the method, which uses such solutions.

The classical theorem, which was established by Briot and Bouquet [10, proves that if the general solution of a polynomial autonomous first order ODE is single-valued, then this solution is either an elliptic function, or a rational function of $e^{\gamma x}, \gamma$ being some constant, or a rational function of $x$. Note that the third case is a degeneracy of the second one, which in its turn is a degeneracy of the first one. It has been proved by Painlevé [1] that the necessary form of the polynomial autonomous first order ODE with the single-valued general solution is

$$
\sum_{k=0}^{m} \sum_{j=0}^{2 m-2 k} h_{j k} y^{j} y_{t}^{k}=0, \quad h_{0 m}=1,
$$

in which $m$ is a positive integer number and $h_{j k}$ are constants.

Rather than to substitute eq. (6) in some nonintegrable system, one can substitute the Laurent series of unknown special solutions in eq. (6) and obtain a system, which is linear in $h_{j k}$ and nonlinear in parameters which are included in the Laurent coefficients.

Let us compare two methods to find special analytic solutions for the generalized Hénon-Heiles system.

The first way is the following:

1) Transform system (2) into eq. (3)

2) Assume that $y$ satisfies some more simple equation, substitute this equation in (3) and obtain a nonlinear algebraic system.

3) Solve the obtained system. 
The second, proposed by R. Conte and M. Musette, way is the following:

1) Choose a positive integer $m$ and define the first order ODE (6), which contains unknown constants $h_{j k}$.

2) Compute coefficients of the Laurent series solutions for (2) with some fixed $C$. The number of coefficients has to be greater than the number of unknowns.

3) Substituting the obtained coefficients, transform eq. (8) in linear and overdetermined system in $h_{j k}$ with coefficients depending on arbitrary parameters.

4) Exclude $h_{j k}$ and obtain the nonlinear system in five parameters.

5) Solve the obtained system.

To obtain the explicit form of the elliptic function, which satisfy the known first order ODE, one can use the classical method due to Poincaré, which has been implemented [1] in Maple [13.

The second way has a few preferences and can be automatized. The first preference is that one does not need to transform system (2) in one differential equation either in $y$ or in $x$. Moreover at $C=-16 / 5$ not $x$, but $x^{2}$ may be an elliptic function. To construct the Laurent series for $x^{2}$ is easier than to find the fourth order equation in $x^{2}$. The main preference of the second method is that the number of unknowns in the resulting algebraic system does not depend on number of coefficients of the first order equation. For example, eq. (6) with $m=8$ includes 60 unknowns $h_{j k}$, and it is not possible use the first way to find similar solutions. Using the second method we obtain nonlinear system in five variables: $\lambda_{1}, \lambda_{2}, H$ and two arbitrary coefficients of the Laurent-series solutions independently of the value of $m$.

The first way also has one important preference. It allows to obtain solutions for an arbitrary $C$, whereas using the second method one has to fix value of $C$ to construct the Laurent series solutions, because of the resonance structure depends on $C$. Using the first way one can also construct equations, which forms are different from (6). For example, the substitution of the eqution

$$
y_{t}^{2}=\tilde{\mathcal{A}}\left(y-P_{0}\right)^{3}+\tilde{\mathcal{B}}\left(y-P_{0}\right)^{5 / 2}+\tilde{\mathcal{C}}\left(y-P_{0}\right)^{2}+\tilde{\mathcal{D}}\left(y-P_{0}\right)^{3 / 2}+\tilde{\mathcal{E}}\left(y-P_{0}\right)
$$

gives two-parameter solutions $[9]$ :

$$
y\left(t-t_{0}\right)=\left(\frac{a \wp\left(t-t_{0}\right)+b}{c \wp\left(t-t_{0}\right)+d}\right)^{2}+P_{0},
$$

where $\wp\left(t-t_{0}\right)$ is the Weierstrass elliptic function, $a, b, c$ and $d$ are some constants. The parameter $P_{0}$ defines the energy of the system. Solutions (8) exist in both above-mentioned nonintegrable cases: $C=-16 / 5$ and $C=-4 / 3$. There exist two different solutions for each pair of values of $C$ and $\lambda$. To find these solutions we a priori assume some class of possible solutions, then compute whether there exists some solution in the given class. We hope that the use of the second method and the corresponding computer algebra program allows to find the three-parameter elliptic solutions without any preliminary assumption. This method is implemented both in REDUCE and in Maple. 


\section{Conclusion}

We have found the special solutions of the Hénon-Heiles system with $C=-16 / 5$ and $C=-4 / 3$ as the Laurent series. For some values of parameters the obtained solutions coincide with the known exact periodic solutions. The Painlevé test does not show any obstacle to the existence of three-parameter single-valued solutions, so, the probability to find exact, for example elliptic, three-parameter solutions, that generalize the solutions found in [9], is high.

The author is grateful to R. Conte, V. F. Edneral, A. K. Pogrebkov and E. I. Timoshkova for valuable discussions. This work has been supported by Russian Federation President's Grants NSh-1685.2003.2 and NSh-1450.2003.2 and by the grant of the scientific Program "Universities of Russia".

\section{References}

1. Painlevé, P.: Leçons sur la théorie analytique des équations différentielles, profeesées à Stockholm (1895) sur l'invitation de S. M. le roi de Suède et de Norwège, Hermann, Paris, 1897. Reprinted in: Oeuvres de P. Painlevé, vol. 1, ed. du CNRS, Paris, 1973. On-line version: The Cornell Library Historical Mathematics Monographs, http://historical.library.cornell.edu/

2. Kowalevski, S.: Sur le problème de la rotation d'un corps solide autour d'un point fixe. Acta Mathematica. 12 (1889) 177-232; Sur une properiété du sustème d'équations différentielles qui définit la rotation d'un corps solide autour d'un point fixe. Acta Mathematica. 14 (1890) 81-93, \{in French\}. Reprinted in: Kovalevskaya, S.V.: Scientific Works, AS USSR Publ. House, Moscow, 1948, \{in Russian\}.

3. Ablowitz M.J., Ramani A., Segur H., A Connection between Nonlinear Evolution Equations and Ordinary Differential Equations of P-type. I \& II, J. Math. Phys. 21 (1980) 715-721 \& 1006-1015

4. Melkonian, S.: Psi-series Solutions of the Cubic Hénon-Heiles System and Their Convergence. J. of Nonlin. Math. Phys. 6 (1999) 139-160, math.DS/9904186.

5. Conte, R., Musette, M.: Analytic solitary waves of nonintegrable equations. Physica D181 (2003) 70, nlin.PS/0302051.

6. Hénon, M., Heiles, C.: The Applicability of the Third Integral of Motion: Some Numerical Experiments, Astronomical J. 69 (1964) 73-79.

7. Conte, R., Musette, M., Verhoeven, C.: Integration of a generalized Hénon-Heiles Hamiltonian, J. Math. Phys. 43 (2002) 1906-1915, nlin.SI/0112030; General solution for Hamiltonians with extended cubic and quartic potensials, TMF (Russ. J. Theor. Math. Phys.) 134 (2003) 148-159, nlin.SI/0301011.

8. Vernov, S.Yu.: Construction of solutions for the generalized Hénon-Heiles system with the help of the Painlevé test. TMF (Russ. J. Theor. Math. Phys.) 135 (2003) 409-419, \{in Russian\}, 792-801, \{in English\}; math-ph/0312048.

9. Vernov, S.Yu., Timoshkova, E.I.: On two nonintegrable cases of the generalized Hénon-Heiles system with an additional nonpolynomial term, math-ph/0402049.

10. Briot, C., Bouquet, T.: Théovie des fonctions doublement périodiques, 1859.

11. van Hoeij, M.: pack. 'algcurves', Maple V (1997), http://www.math.fsu.edu/

12. Hearn, A.C.: REDUCE. User's and Contributed Packages Manual, Vers. 3.7, CA and Codemist Ltd, Santa Monica, California, 1999, http://www.zib.de/Symbolik/reduce/more/moredocs/reduce.pdf

13. Heck, A.: Introduction to Maple, 3rd Edition, Springer-Verlag, New York, 2003. 